Article

\title{
Nanoporous Gold Nanocomposites as a Versatile Platform for Plasmonic Engineering and Sensing
}

\author{
Fusheng Zhao ${ }^{1}$, Jianbo Zeng ${ }^{1}$ and Wei-Chuan Shih ${ }^{1,2,3,4,5, *}$ \\ 1 Department of Electrical and Computer Engineering, University of Houston, 4800 Calhoun Rd, Houston, \\ TX 77004, USA; fzhao3@uh.edu (F.Z.); jzeng8@uh.edu (J.Z.) \\ 2 Department of Biomedical Engineering, University of Houston, 4800 Calhoun Rd, Houston, TX 77004, USA \\ 3 Program of Materials Science and Engineering, University of Houston, 4800 Calhoun Rd, Houston, \\ TX77004, USA \\ 4 Department of Chemistry, University of Houston, 4800 Calhoun Rd, Houston, TX 77004, USA \\ 5 Biomedical Institute for Global Health Research and Technology (BIGHEART), National University of \\ Singapore 14 Medical Drive, Singapore 117599, Singapore \\ * Correspondence: Wshih@uh.edu; Tel.: +1-713-743-4454
}

Received: 1 June 2017; Accepted: 24 June 2017; Published: 28 June 2017

\begin{abstract}
Plasmonic metal nanostructures have shown great potential in sensing applications. Among various materials and structures, monolithic nanoporous gold disks (NPGD) have several unique features such as three-dimensional (3D) porous network, large surface area, tunable plasmonic resonance, high-density hot-spots, and excellent architectural integrity and environmental stability. They exhibit a great potential in surface-enhanced spectroscopy, photothermal conversion, and plasmonic sensing. In this work, interactions between smaller colloidal gold nanoparticles (AuNP) and individual NPGDs are studied. Specifically, colloidal gold nanoparticles with different sizes are loaded onto NPGD substrates to form NPG hybrid nanocomposites with tunable plasmonic resonance peaks in the near-infrared spectral range. Newly formed plasmonic hot-spots due to the coupling between individual nanoparticles and NPG disk have been identified in the nanocomposites, which have been experimentally studied using extinction and surface-enhanced Raman scattering. Numerical modeling and simulations have been employed to further unravel various coupling scenarios between AuNP and NPGDs.
\end{abstract}

Keywords: nanoporous gold disk; nanoporous nanocomposite; surface-enhanced Raman scattering; plasmonic sensing

\section{Introduction}

In recent years, the intriguing optical properties of metallic nanostructures have become a research focus. Light-excited collective oscillation of conduction-band electrons in metallic nanostructures is known as surface plasmon polariton (SPP) for propagating ones and localized surface plasmon resonance (LSPR) for non-propagating ones. Both SPP and LSPR exhibit a significant dependence on the near-field environment in the close proximity to the nanostructures. Local changes in the dielectric function can significantly modulate the resonance frequency, which has become an effective means for molecular sensing [1-3]. For example, the proximity influence of target molecules can be considered as a slight increase in the local refractive index against the original index in either the air or water. When the proximity entity is another metal nanostructure or nanoparticle, plasmonic coupling can occur and causes pronounced modulation in the combined optical properties. Plasmonic coupling between nanostructures can lead to resonance frequency shifts, plasmonic hybridization, "hot-spots" generation, changes in radiation damping, etc. [4-8]. From the design and optimization point of view, plasmonic coupling can provide an alternative means for plasmonic engineering and sensing. Plasmon-induced 
electric field (E-field) localization has been recognized as the primary mechanism in surface-enhanced spectroscopy, such as surface-enhanced Raman scattering (SERS) $[9,10]$, surface-enhanced fluorescence (SEF) [11], surface-enhanced infrared absorption (SEIRA) [12,13], and surface-enhanced near-infrared absorption (SENIRA) [14].

The performance of surface plasmon-based techniques depends on a well-designed plasmonic substrate with desirable plasmonic properties. For instance, photothermal therapy requires the resonance peak position to be inside the near-infrared region, also known as the "diagnostic/therapeutic window", for deeper tissue penetration [15]. The performance of surface-enhanced spectroscopy is typically better when the LSPR resonance aligns with the excitation and/or the emission/scattering wavelengths [16]. In addition to placing the LSPR resonance at the desired wavelength, plasmonic coupling-induced "hot-spots" provide additional E-field enhancement to further intensify light-matter interactions [17-20]. The coupling between closely located nanoparticles was shown to be efficient for both purposes: the near-field coupling can effectively shift the LSPR peak position [21] and the nano-gaps between particles can generate strong E-field due to gap-mode resonance [22,23].

Lithographically patterned sub-micron nanoporous gold disk (NPGD) features large surface area and high-density hot-spots [24]. It has been demonstrated in DNA cancer marker detection by both SERS and SEF $[25,26]$, label-free sensing and imaging of physiological small analytes for disease diagnosis [27], photothermal inactivation of pathogens [28,29], and chemical analysis by SENIRA [14]. In addition to providing highly enhanced spectroscopy sensing capabilities, NPGDs also have plasmonic properties that can be tuned by varying their external geometrical features via lithographic patterning and internal nanoporous morphology via controlled dealloying [30], laser and furnace annealing [30,31], and surface modifications [32].

In this work, we demonstrate a versatile platform for plasmonic engineering and sensing by loading colloidal nanoparticles onto NPGD. Gold nanoparticles (AuNP) with different sizes are loaded onto NPGD substrates to form hybrid nanocomposites, which exhibit red-shifted resonance peaks compared to bare NPGD. From the material design aspect, the magnitude of the coupling-induced shift is larger than other approaches described previously [30-32]. The plasmonic resonance is tunable in the near infrared (NIR) spectral region by changing the AuNP size. Finite-difference time-domain (FDTD) simulation reveals that coupling-induced hot-spots not only originate from the interaction between NPGD and AuNP, but also from between AuNPs. From the sensing aspect, the platform can be employed to detect AuNP when they are in the proximity. In addition, potential applications of nanocomposites in molecular sensing by SERS will be illustrated using 3,3'-diethylthiatricarbocyanine iodide (DTTC) molecule. The results suggest that the nanocomposites exhibit improved SERS sensitivity and can be used as novel sensors and imaging labels [33].

\section{Materials and Methods}

\subsection{Materials}

Chloroform (anhydrous, $\geq 99.0 \%$ ), nitric acid (ACS reagent, 70\%), poly(diallyldimethylammonium chloride) (PDDA, 20 wt. \% in $\mathrm{H}_{2} \mathrm{O}$ ), 3,3'-diethylthiatricarbocyanine iodide (DTTC, 99\%), sodium citrate dehydrate ( $\geq 99.0 \%$ ), gold (III) chloride hydrate (99.999\% trace metals basis), sodium dodecyl sulfate (ACS reagent, $\geq 99.0 \%$ ) and latex beads (polystyrene beads (PS beads), 10\% aqueous suspension) with mean particle sizes $460 \mathrm{~nm}$ were purchased from Sigma-Aldrich. Ethanol (200 proof) was from Decon Laboratories, Inc. Silicon wafers were obtained from University Wafers, and coverglass $(22 \mathrm{~mm} \times$ $40 \mathrm{~mm}$, No. 1) from VWR. $\mathrm{Ag}_{70} \mathrm{Au}_{30}$ (atomic percentage) alloy sputtering targets were purchased from ACI Alloys, Inc. Argon gas (99.999\%) was used for RF-sputter etching; $50 \mathrm{~nm}$ AuNP were purchased from BBI Solutions. 


\subsection{Characterization}

Scanning electron microscope (SEM) images were obtained from PHILIPS FEI XL-30 FEG-SEM system. A Cary 50 Scan UV-visible spectrometer and a Jasco V-570 UV-Vis-NIR spectrophotometer were used to measure extinction spectra of the monolayer NPGDs and nanocomposites on a glass coverslip. The SERS spectra of DTTC were recorded by using a home-built line-scan Raman microscope, and the automated image curvature correction algorithm was employed, followed by 5th-order polynomial background removal [34].

\subsection{Fabrication of Nanoporous Composites}

Several fabrication methods for NPG nanoparticles have been reported in the literature; these methods are based on dewetting process, [35,36] nanosphere lithography technique, [24] and electron-bean lithography (EBL) technique [37]. The dewetting process-based fabrication method produces NPG nanoparticles highly irregular in shape and size, whereas the latter two produce well-controlled nanoparticles. With nanosphere lithography-based fabrication process, a monolayer of polystyrene micron-beads is used as etching template for the patterning of silver-gold alloy nanodisks which are further processed into NPG nanoparticle through dealloying process [24]. Such a method produces monodispersed monolithic NPG nanodisk high-density arrays as shown in Figure 1a. The EBL-based method provides even better control over the nanoparticle shape, size and locations. With such a technique, the nanoparticles are fabricated by EBL and lift-off process. The silver-gold alloy composition for the precursor nanoparticle is achieved by the evaporation of alternating silver and gold thin layers followed by thermal annealing. This method is capable of fabricating arbitrary-shaped NPG nanoparticle (Figure 1b-e) with a predetermined location such as periodic arrays or random arrays (Figure 1f,g).

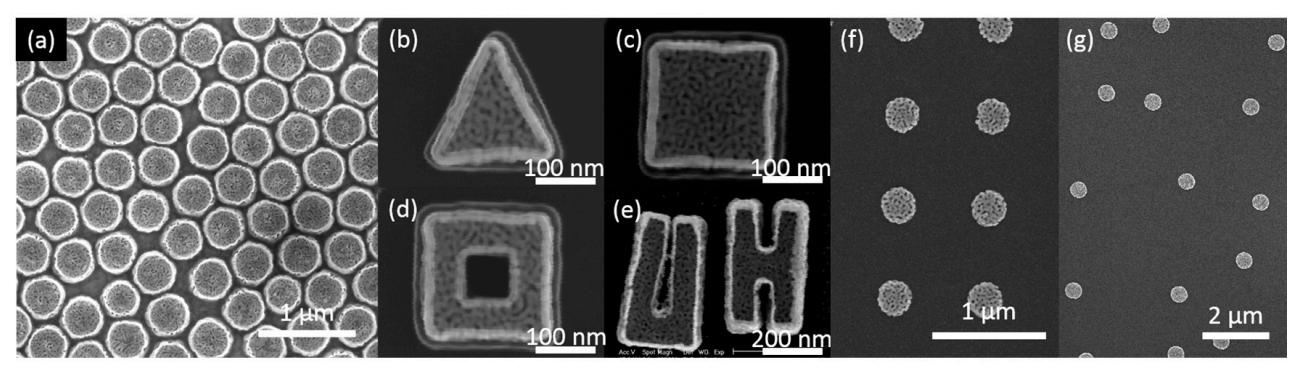

Figure 1. SEMs of nanoporous gold (NPG) nanoparticles fabricated by various methods. (a) nanoporous gold disk (NPGD) array fabricated by a method based on nanosphere lithography; (b-e) Arbitrary NPG nanoparticles; (f) Periodic NPGD array; and (g) Random NPGD array fabricated by a method based on electron-bean lithography (EBL).

In this study, the NPGD are fabricated through nanosphere lithography-based method. Fabrication conditions produce as-prepared NPGDs with $\sim 300 \mathrm{~nm}$ diameter, $\sim 75 \mathrm{~nm}$ thickness and $\sim 8.5 \mathrm{~nm}$ pore size on average. The NPG nanocomposites were synthesized by taking advantage of the electrostatic interaction mechanism that occurs between negatively charged AuNPs and positively charged NPGD surfaces. Such a method provides an easy route for the attachment of AuNPs compared with covalent attachment, since the molecules used for positively charging the NPGD substrate are readily available. As shown in Figure 2, NPGDs were functionalized with PDDA to achieve positively charged surfaces. Negatively charged AuNPs with $\sim 13 \mathrm{~nm}$ and $\sim 50 \mathrm{~nm}$ mean diameter with $<10 \%$ coefficient of variation were prepared by Turkevich protocol [38,39], or purchased from BBI Solutions, respectively. AuNPs were washed one time by centrifugation to remove negatively charged surfactants and concentrated 10 times. Positively charged NPGDs were then incubated in negatively charged AuNPs colloidal solutions for $\sim 15 \mathrm{~h}$ to form nanocomposites. After incubation, the samples were thoroughly washed with deionized (DI) water and subsequently dried by nitrogen flow. 


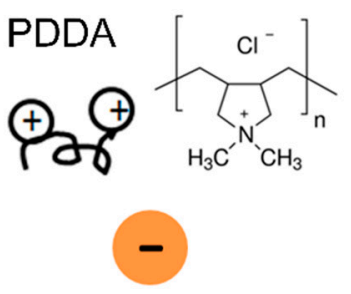

AuNP

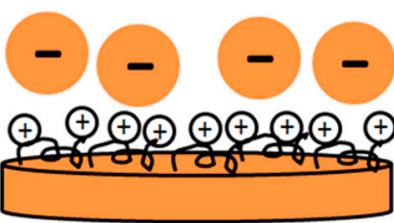

NPGD/PDDA

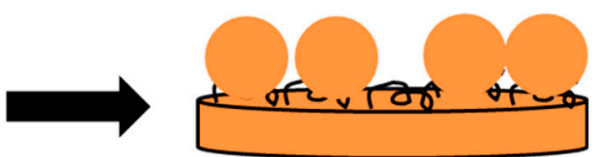

NPGD/AuNP

Figure 2. The strategy to synthesize NPG nanocomposites.

\section{Results and Discussion}

\subsection{Characterization of Nanoporous Composites}

SEM images of NPGD/AuNP composites are shown in Figure 3 where $13 \mathrm{~nm}$ AuNPs were employed in (a) and (b) and $50 \mathrm{~nm}$ AuNPs in (c) and (d). For a fair comparison, it is desired that AuNPs of both sizes are attached on the surfaces of NPGDs instead of loaded into the pores. Therefore, the alloy (Ag70at.\%Au30at.\%) disks were dealloyed in 68\% nitric acid for $1 \mathrm{~min}$ to obtain an average pore size of $\sim 8.5 \mathrm{~nm}$. As shown in Figure 3a, $13 \mathrm{~nm}$ AuNPs attached to NPGDs with a higher density and formed structures such as dimers, trimers, tetramers and assembled clusters. The particle density of attached $50 \mathrm{~nm}$ AuNPs on NPGDs (Figure 3c) is much lower than that of the $13 \mathrm{~nm}$ (Figure 3a). At a low magnification (Figure 3b,d), both SEM images show the inherent spatial heterogeneity of AuNPs on NPGDs due to the random attaching process.

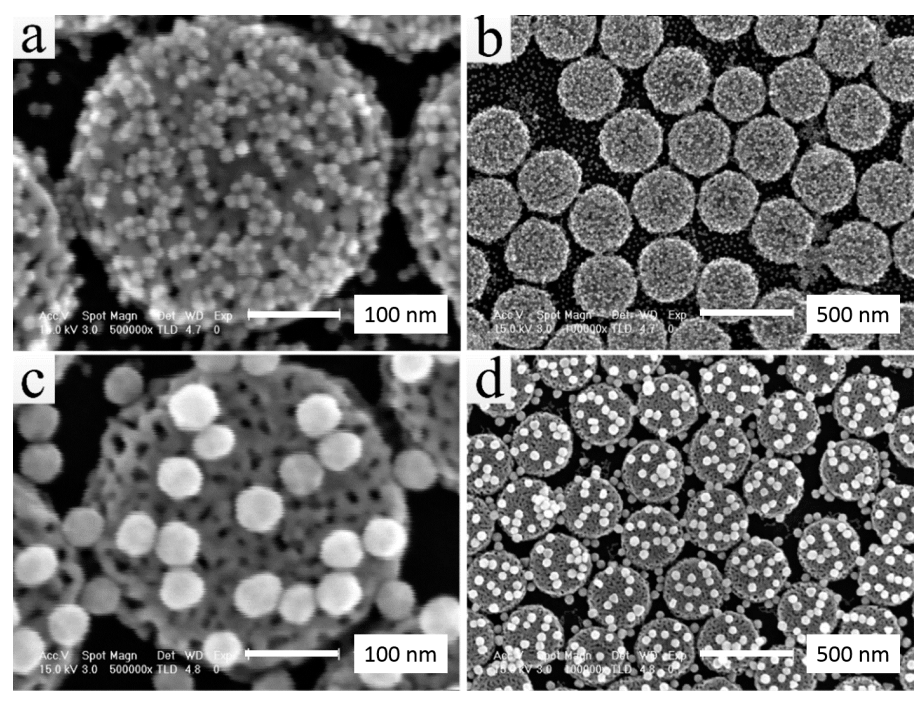

Figure 3. SEM images of NPGD/AuNP composites: (a) and (b) $13 \mathrm{~nm}$ AuNPs on NPGD; (c) and (d) $\sim 50 \mathrm{~nm}$ AuNPs on NPGDs. AuNP: gold nanoparticles.

The plasmonic properties of NPGD/AuNP composites were investigated in comparison with AuNPs and NPGDs (Figure 4). As shown in Figure 4a, $13 \mathrm{~nm}$ and $50 \mathrm{~nm}$ AuNPs in water exhibit extinction peaks at 524 and $530 \mathrm{~nm}$, respectively [40]. NPGD with $300 \mathrm{~nm}$ diameter has the extinction peak at $960 \mathrm{~nm}$ in air (Figure 4b), which corresponds to the in-plane dipole resonance mode. The peak position red-shifted to $1067 \mathrm{~nm}$ when the NPGDs are attached with $13 \mathrm{~nm}$ AuNPs, and red-shifted to $1152 \mathrm{~nm}$ upon attaching $50 \mathrm{~nm}$ AuNPs. It is noted that the plasmon resonance of NPGD is sensitive to the refractive index changes [24]. Therefore, the peak shift caused by adsorbed PDDA was investigated. As shown in Figure S1, the extinction peak of NPGD red-shifted $\sim 15 \mathrm{~nm}$ after it was functionalized with PDDA. Apparently, the much stronger red-shifts induced by the adsorption 
of AuNPs originated from the interaction between NPGDs and AuNPs. In our previous work [24], we reported that the strong plasmonic coupling generated between the nanoporous structures and the patterned disk shape significantly red-shifts the in-plane dipole resonance peak. On the other hand, a decrease of the plasmonic coupling caused by the increased pore and ligament size induces blue-shift [30]. Qian et al. demonstrated a giant SERS enhancement primarily due to strong coupling between nanopore and AuNPs, which was further confirmed by FDTD simulation [41]. In this work, the red-shifts of NPGD/AuNP composite extinction peaks also indicate a strong plasmonic coupling between AuNPs and NPGDs. The interaction between NPGDs and $13 \mathrm{~nm}$ AuNPs induced $\sim 92 \mathrm{~nm}$ red-shift of the extinction peak, whereas $50 \mathrm{~nm}$ AuNPs induced $177 \mathrm{~nm}$ red-shift. To our knowledge, this is the first study to demonstrate the effect of AuNP size on its coupling strength with plasmonic nanoporous materials.
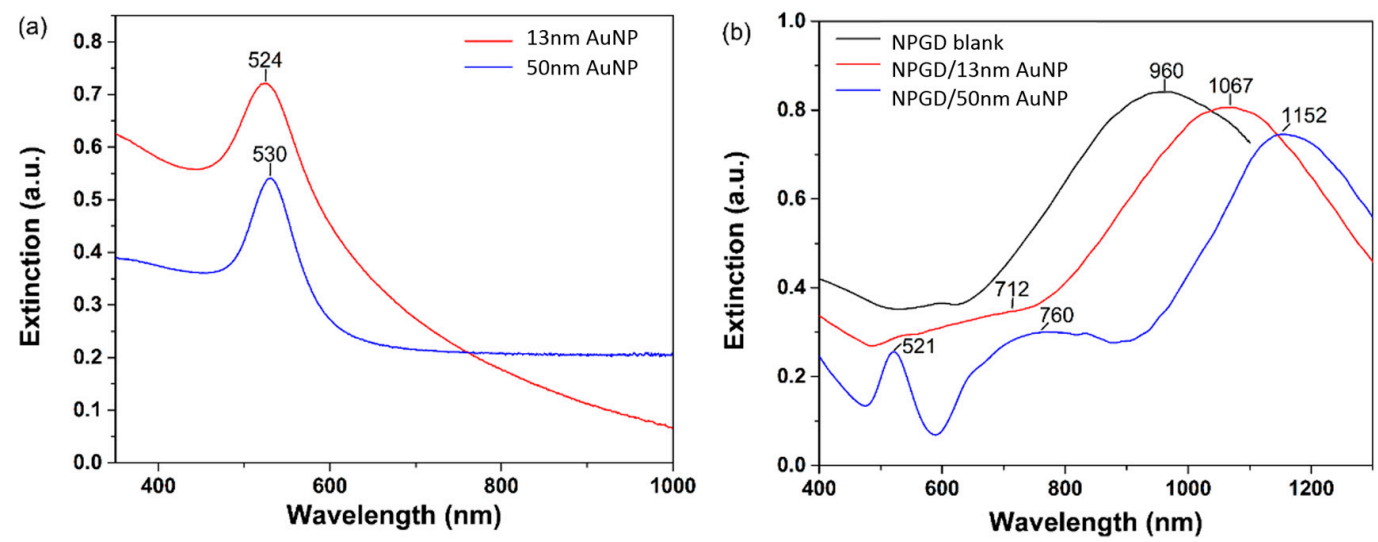

Figure 4. Extinction spectra: (a) AuNPs; and (b) NPGD/AuNP composites.

To further elucidate the red-shift behavior of AuNP on NPGD, we performed FDTD simulations. The 3D NPGD structure used in the simulation is generated using 2D SEM images. As shown in Figure $5 \mathrm{a}$, the simulated LSPR peak for NPGD is at $990 \mathrm{~nm}$, NPGD/13 nm AuNP composite at $1030 \mathrm{~nm}$, and NPGD/50 nm AuNP composite at $1150 \mathrm{~nm}$. The trend of the peak red-shift is consistent with experimental results. The extinction spectra of NPGD/AuNP composites suggest that the loading of AuNPs could markedly influence the LSPR of NPGD in the NIR region. To show such red-shift is indeed caused by coupling between NPGD and AuNP, similar simulations were carried out with AuNPs replaced by PS beads. As shown in Figure 5b, the PS beads-induced refractive index increase had limited effect on the peak position of the LSPR of NPGD.
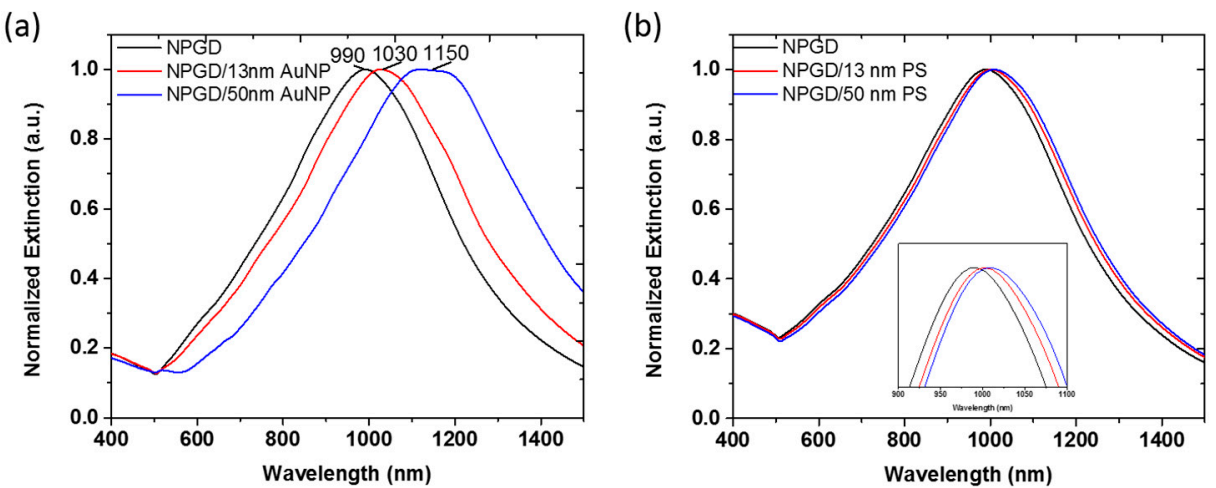

Figure 5. FDTD-simulated extinction spectra. (a) $300 \mathrm{~nm}$ blank NPGD, NPGD/13 nm AuNP composite and NPGD/50 nm AuNP composite; (b) Same setups as (a) with AuNP replaced by polystyrene (PS) beads of the same size. FDTD: finite-difference time-domain. 
The E-field distributions of the nanocomposites were also simulated with FDTD as shown in Figure 6. In comparing the simulation results at $785 \mathrm{~nm}$ (Figure 6a-e) with the ones obtained at the resonance wavelength (Figure $6 \mathrm{f}-\mathrm{j}$ ), the E-field is stronger for the latter ones, as expected. Also, it is clear that the hot-spots are more concentrated at the disk center with $785 \mathrm{~nm}$ wavelength, whereas in the case of resonance wavelength, the hot-spots have moved to the left and right edges of the disk. This is simply due to the fact that the dominating LSPR resonance mode is the dipolar disk mode resonance, and thus the mode shape.

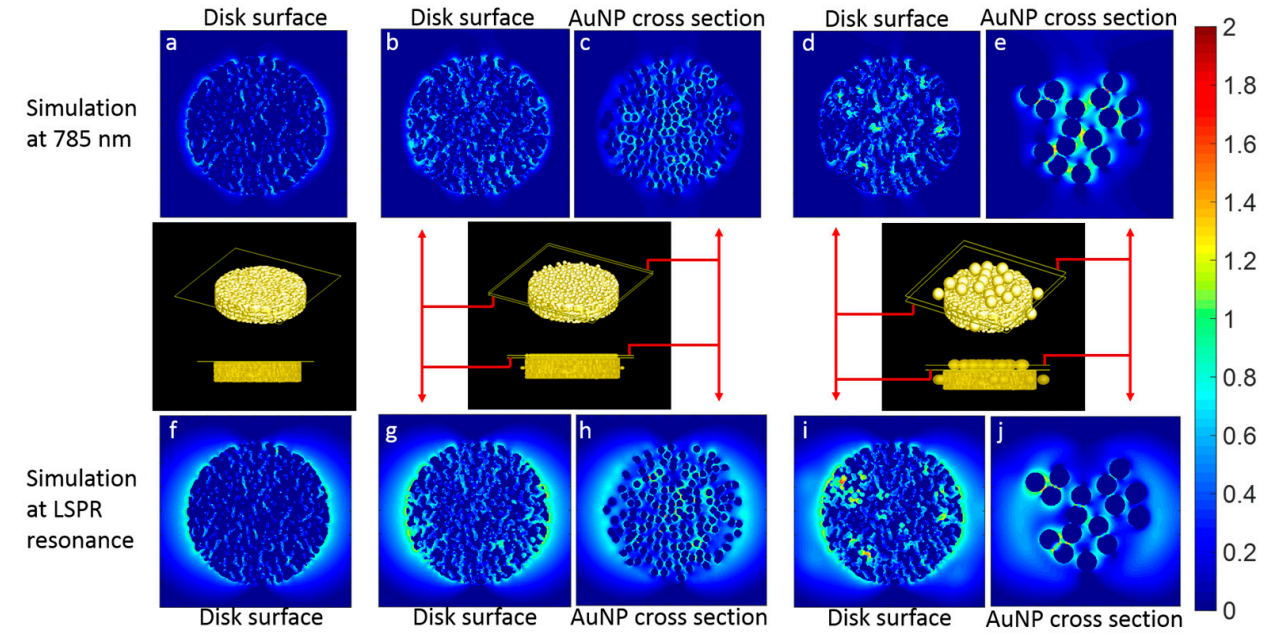

Figure 6. FDTD simulation results of E-field distribution: (a,f) blank NPGD; $(\mathbf{b}, \mathbf{c}, \mathbf{g}, \mathbf{h})$ NPGD/13 nm AuNP composite; and (d,e,i,j) NPGD/50 nm AuNP composite. The E-field distributions are calculated for both the excitation laser wavelength $(785 \mathrm{~nm}, \mathrm{a}-\mathrm{e})$ used in SERS measurements and the LSPR wavelength for various nanocomposites: NPGD (990 nm, f), NPGD/13 nm AuNP (1030 nm, g,h) and NPGD/50 nm AuNP (1150 nm, i,j). The scale bar is the log10 of the relative E-field intensity.

Furthermore, by comparing the E-field distribution of the NPGD and NPGD/AuNP composites, it is readily seen that additional hot-spots exist in the composites. The additional hot-spots at the NPGD surface indicate a strong coupling between NPGD and AuNP, whereas the hot-spots between AuNPs indicate a strong coupling among AuNPs.

Finally, by comparing the E-field distribution of the NPGD/13 nm AuNP composites with the NPGD/50 nm AuNP composites, it is observed that the $13 \mathrm{~nm}$ AuNP generated a higher number of hot-spots due to the higher particle density, whereas the $50 \mathrm{~nm}$ AuNP has a lower number of hot-spots but stronger intensity due to a stronger coupling. The stronger coupling for $50 \mathrm{~nm}$ AuNP corresponds well with the stronger red-shift in the extinction spectra.

Another set of simulations were conducted for a single $50 \mathrm{~nm}$ AuNP located on or near a NPGD at different positions. Such simulations reveal the fact that the coupling strength and the amount of peak shift induced by nanoparticles are highly location dependent. The simulated center positions of the $50 \mathrm{~nm}$ AuNP are indicated in Figure 7a. When the center is within the NPGD boundary, the AuNP is located above the NPGD with $2 \mathrm{~nm}$ separation, otherwise, the AuNP is located parallel to the NPGD. The amount of peak shifts induced by the single AuNP at different locations are analyzed and plotted into a colormap as shown in Figure 7b. The colormap corresponds well with the E-field distribution of the NPGD at its resonance wavelength. That is, when the AuNP is located inside the enhanced E-field generated by NPGD, strong coupling effect can occur. The hot-spots induced by single AuNP at different locations are also being investigated. The E-field at six locations along the polarization direction ranging from the center to out of the NPGD boundary are plotted in Figure 7c. These figures clearly show a higher E-field enhancement when the AuNP is located near the edge of the NPGD, which corresponds well with the stronger coupling observed earlier. The results shown 
here clearly indicated that the AuNPs located close to the edges contribute more to the peak shift and hot-spots generation. Notice that, here the hot-spots are generated between AuNP and NPGD; the hot-spots generated between AuNPs should not be affected by the particle position.

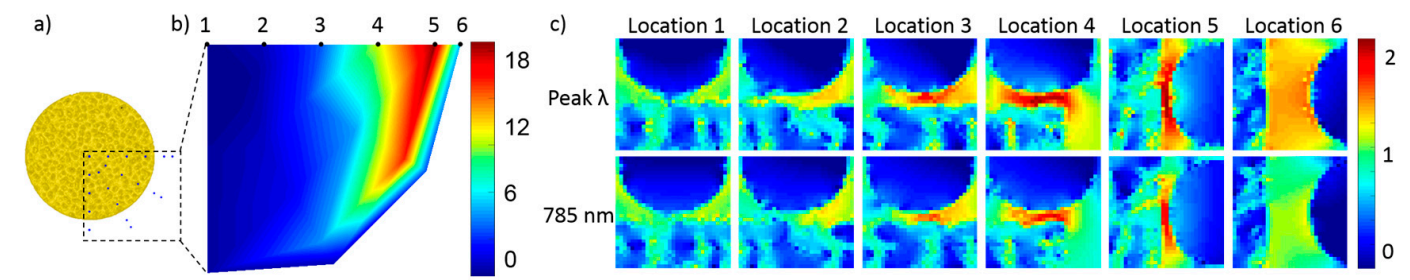

Figure 7. Simulation result for coupling between a single NPGD and a single AuNP. The locations of the single AuNP with respect to the NPGD are indicated in (a); the peak shift induced by the AuNP at different locations are plotted as a colormap in (b); and (c) the E-field distribution between the AuNP and the NPGD at location 1 to 6 indicated in (b); The scale bar is the $\log 10$ of the relative E-field intensity.

\subsection{Probing Hot-Spots by Surface Adsorbates}

SERS has been an effective means to probe plasmonic hot-spots in AuNP oligomers. Compared with AuNPs, NPGDs not only possess large surface area but also high-density hot-spots, which are proven to provide better sensing performance. Herein, near-infrared dye 3,3'-diethylthiatricarbocyanine (DTTC) is used as a probing molecule for assessing hot-spots. The typical SERS spectrum of DTTC on NPGDs is shown in Figure 8a (black trace) with prominent Raman peaks at frequencies such as 1240,1131,1078, 845,779, 627 and $500 \mathrm{~cm}^{-1}$. The peak at $1240 \mathrm{~cm}^{-1}$ is assigned to C-N-C symmetrical stretching, while the strong shoulder peak at $1078 \mathrm{~cm}^{-1}$ originates from C-C-C symmetrical stretching [42]. The intense Raman peaks at 1131, and 845 and $627 \mathrm{~cm}^{-1}$ belong to $\mathrm{C}-\mathrm{C}$ stretching of various $\mathrm{C}-\mathrm{C}$ bonds in the molecular skeleton. The detailed assignments based on density functional theory simulation were reported by Cai and coworkers [42].

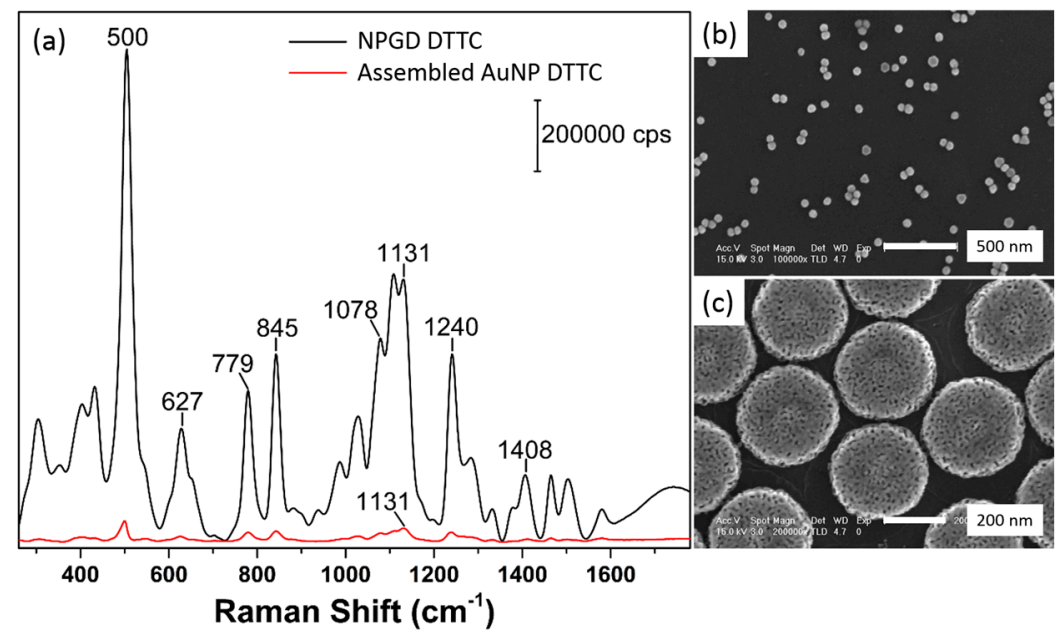

Figure 8. (a) SERS spectra of DTTC on NPGD and $50 \mathrm{~nm}$ AuNPs; (b) SEM image of $50 \mathrm{~nm}$ AuNPs assembled on Si wafer, scale bar $500 \mathrm{~nm}$; (c) SEM image of $300 \mathrm{~nm}$ NPGD on Si wafer, scale bar $200 \mathrm{~nm}$. SERS: surface-enhanced Raman scattering; DTTC: 3,3'-diethylthiatricarbocyanine iodide.

We first compare SERS spectra of DTTC adsorbed on NPGDs and $50 \mathrm{~nm}$ AuNPs (Figure 8a). The corresponding SEM images of self-assembled AuNPs and as-prepared NPGDs are shown in Figure 8b,c, respectively. As shown in Figure 8b, $50 \mathrm{~nm}$ AuNPs were assembled onto PDDA-modified Si 
wafer. The density of assembled AuNPs could be estimated based on the SEM image to be 27 particles per $\mu \mathrm{m}^{2}$, while the density of NPGDs is $\sim 9$ particles per $\mu \mathrm{m}^{2}$. Since SERS spectra of both nanostructures were measured at the same conditions, the estimated Raman intensity of a single NPGD at $1131 \mathrm{~cm}^{-1}$ is $\sim 60$ times stronger than that of a single $50 \mathrm{~nm}$ AuNP. We also estimate the number of adsorbed DTTC molecules on NPGD and AuNP. First, a calibration curve was established by a linear fitting of DTTC concentrations versus absorbance intensity at $763 \mathrm{~nm}$ from $10^{-8}$ to $10^{-5} \mathrm{M}$ (Figure 9). As shown in Figure $9 \mathrm{~b}$, the $\mathrm{R}^{2}$ value of the calibration curve is 0.9997 which indicates high-quality fit. NPGDs samples $(\sim 1 \mathrm{~cm} \times 1 \mathrm{~cm})$ were placed at the bottom of a cuvette for 1-h incubation, and the in situ absorption spectra of the solution were recorded every $20 \mathrm{~min}$. The adsorption of DTTC molecules at NPGD surfaces decreases the intensity of DTTC absorbance peak. The number of adsorbed DTTC molecules is then estimated through the linear fitting equation, which is $\sim 460,000$ DTTC molecules per NPGD after incubating $1 \mathrm{~h}$ in $10^{-5} \mathrm{M}$ solution. On the other hand, the capability of AuNP to adsorb DTTC molecules is limited by its geometrical structure, where only $\sim 26,000$ molecules per particle could be adsorbed based on calculation.
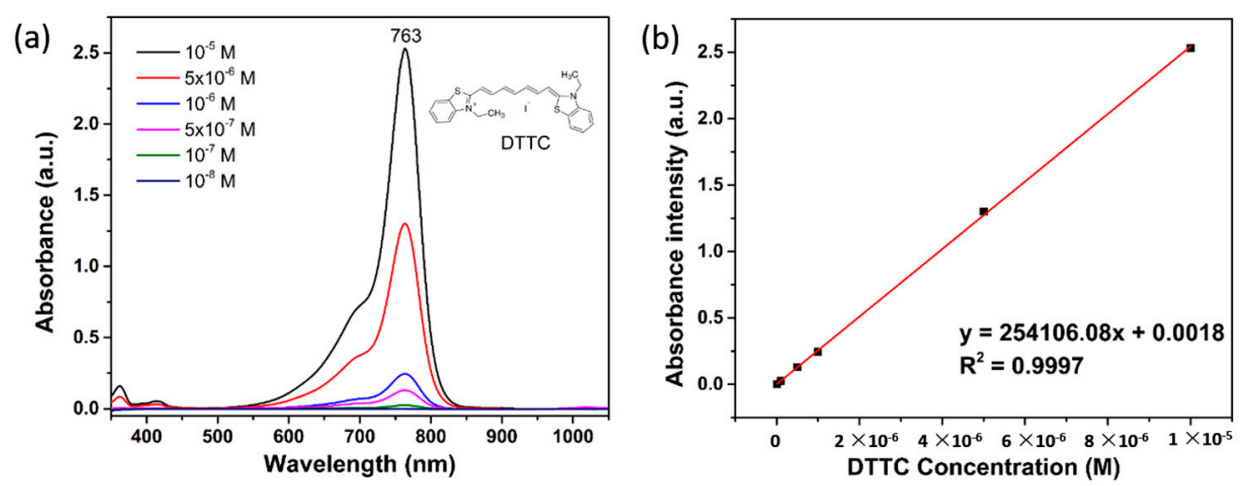

Figure 9. (a) UV-vis absorbance spectra of DTTC at different concentrations; (b) Linear fitting of DTTC concentration vs. absorbance intensity.

The SERS spectra of DTTC loaded on NPGD/50 nm AuNP composites are shown in Figure 10a, and the spectra obtained from NPGD/13 nm AuNP composites are shown in Figure S2. The four spectra represent different DTTC loading strategies: NPGD loaded with DTTC as control, NPGD/50 nm AuNP composites loaded with DTTC, NPGD loaded with DTTC and then assembled with $50 \mathrm{~nm}$ AuNPs, and NPGD and $50 \mathrm{~nm}$ AuNP loaded with DTTC with the same protocols separately and then assembled together.

(a)

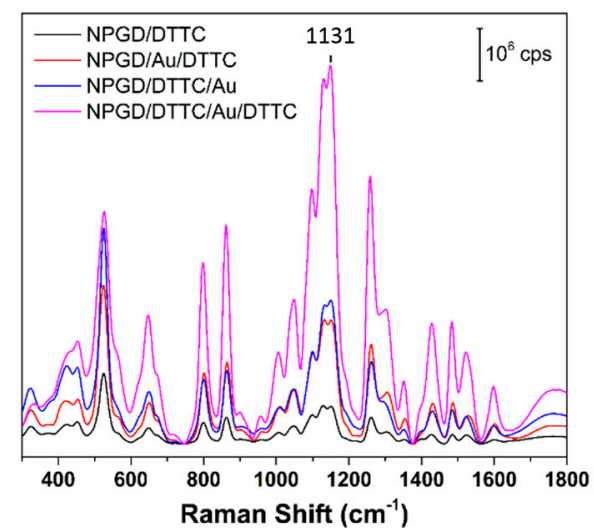

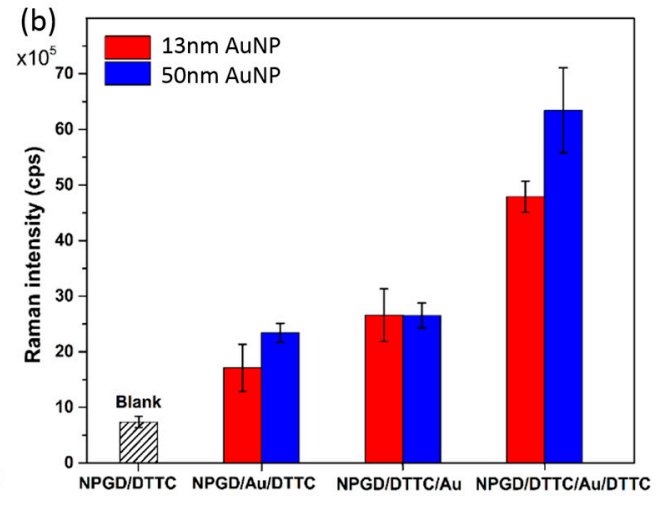

Figure 10. (a) SERS spectra of DTTC on NPGD/50 nm AuNP composites; (b) SERS performance comparison between 13 and $50 \mathrm{~nm}$ AuNPs on NPGDs. 
The $1131 \mathrm{~cm}^{-1}$ peak is used for quantitative evaluation. The SERS performance of $13 \mathrm{~nm}$ and $50 \mathrm{~nm}$ AuNPs on NPGDs are shown in Figure 10b. For both $13 \mathrm{~nm}$ and $50 \mathrm{~nm}$ AuNPs on NPGDs, the SERS peaks have the following relationship: NPGD/DTTC $<$ NPGD/Au/DTTC $<$ NPGD/DTTC/Au $<$ NPGD/DTTC/Au/DTTC. The Raman intensity of NPGD/Au/DTTC has significantly increased compared to NPGD/DTTC. This is due to the additional hot-spots generated by assembled AuNPs. Loading DTTC before assembling AuNPs on NPGD (NPGD/DTTC/Au) resulted in higher intensity than loading DTTC after the assembly of AuNPs. The possible reason is that in the former loading strategy, more DTTC molecules are adsorbed at the gaps between the NPGD surface and the assembled AuNPs, where the hot-spots are the strongest (according to Figure 6). It is easy to understand that the last loading strategy has the strongest Raman intensity, as this method allows adsorbed DTTC molecules to occupy most of the hot-spots.

Finally, to illustrate the size effect of AuNPs in SERS performance, we now compare the Raman intensity of nanocomposites made from 13 and $50 \mathrm{~nm}$ AuNPs. Under the same DTTC loading strategy, NPGD/50 nm AuNP generally has better performance, mainly due to the existence of stronger hot-spots in such nanocomposites as shown in Figure 6.

\section{Conclusions}

In this paper, hybrid nanoporous gold composites have been developed for plasmonic engineering by loading AuNPs of different sizes onto NPGD array substrates. Due to coupling, the hybrid nanocomposites have red-shifted resonance peaks compared to bare NPGD array substrates. The plasmonic resonance is tunable in the near-infrared spectral range by changing the AuNP size. FDTD simulation correlates well with the experimental results, where larger AuNPs generated stronger E-field hot-spots and a stronger red-shift. The simulation also reveals that the coupling-induced hot-spots not only originate from the interactions between NPGD and AuNP, but also from between AuNPs. Hot-spots formation on various entities have been experimentally studied by surface-enhanced Raman scattering of adsorbates.

Supplementary Materials: The following are available online at http:/ /www.mdpi.com/1424-8220/17/7/1519/ s1, Figure S1: Extinction spectra of NPGD before and after adsorbing PDDA. Figure S2: SERS spectra of DTTC on NPGD/13 nm AuNP composites.

Acknowledgments: This work was partially funded by National Science Foundation (NSF) CAREER Award-1151154 and NSF 1605683.

Author Contributions: F.Z., J.Z. and W.C.S. conceived and designed the experiments; F.Z. and J.Z. performed the experiments; F.Z., J.Z. and W.C.S. analyzed the data; F.Z. performed the simulation; F.Z., J.Z. and W.C.S. wrote the paper.

Conflicts of Interest: The authors declare no conflict of interest.

\section{References}

1. Willets, K.A.; Van Duyne, R.P. Localized surface plasmon resonance spectroscopy and sensing. Ann. Rev. Phys. Chem. 2007, 58, 267-297. [CrossRef] [PubMed]

2. Swiontek, S.E.; Pulsifer, D.P.; Lakhtakia, A. Optical sensing of analytes in aqueous solutions with a multiple surface-plasmon-polariton-wave platform. Sci. Rep. 2013, 3, 1409. [CrossRef]

3. Zeng, S.; Baillargeat, D.; Ho, H.-P.; Yong, K.-T. Nanomaterials enhanced surface plasmon resonance for biological and chemical sensing applications. Chem. Soc. Rev. 2014, 43, 3426-3452. [CrossRef] [PubMed]

4. Fischer, H.; Martin, O.J. Retardation-induced plasmonic blinking in coupled nanoparticles. Opt. Lett. 2009, 34, 368-370. [CrossRef] [PubMed]

5. Prodan, E.; Radloff, C.; Halas, N.J.; Nordlander, P. A hybridization model for the plasmon response of complex nanostructures. Science 2003, 302, 419-422. [CrossRef] [PubMed]

6. Kravets, V.G.; Schedin, F.; Grigorenko, A.N. Extremely narrow plasmon resonances based on diffraction coupling of localized plasmons in arrays of metallic nanoparticles. Phys. Rev. Lett. 2008, 101, 087403. [CrossRef] [PubMed] 
7. Nie, S.; Emory, S.R. Probing single molecules and single nanoparticles by surface-enhanced raman scattering. Science 1997, 275, 1102-1106. [CrossRef] [PubMed]

8. Humphrey, A.D.; Barnes, W.L. Plasmonic surface lattice resonances on arrays of different lattice symmetry. Phys. Rev. B 2014, 90. [CrossRef]

9. Stiles, P.L.; Dieringer, J.A.; Shah, N.C.; Van Duyne, R.P. Surface-enhanced raman spectroscopy. Annu. Rev. Anal. Chem. 2008, 1, 601-626. [CrossRef] [PubMed]

10. Thacker, V.V.; Herrmann, L.O.; Sigle, D.O.; Zhang, T.; Liedl, T.; Baumberg, J.J.; Keyser, U.F. DNA origami based assembly of gold nanoparticle dimers for surface-enhanced raman scattering. Nat. Commun. 2014, 5. [CrossRef] [PubMed]

11. Fort, E.; Grésillon, S. Surface enhanced fluorescence. J. Phys. D Appl. Phys. 2008, 41, 013001. [CrossRef]

12. Osawa, M. Dynamic processes in electrochemical reactions studied by surface-enhanced infrared absorption spectroscopy (SEIRAS). B Chem. Soc. Jpn. 1997, 70, 2861-2880. [CrossRef]

13. Huck, C.; Neubrech, F.; Vogt, J.; Toma, A.; Gerbert, D.; Katzmann, J.; Härtling, T.; Pucci, A. Surface-enhanced infrared spectroscopy using nanometer-sized gaps. ACS Nano 2014, 8, 4908-4914. [CrossRef] [PubMed]

14. Shih, W.C.; Santos, G.M.; Zhao, F.; Zenasni, O.; Arnob, M.M. Simultaneous chemical and refractive index sensing in the 1-2.5 mum near-infrared wavelength range on nanoporous gold disks. Nano Lett. 2016, 16, 4641-4647. [CrossRef] [PubMed]

15. Huang, X.H.; El-Sayed, I.H.; Qian, W.; El-Sayed, M.A. Cancer cell imaging and photothermal therapy in the near-infrared region by using gold nanorods. J. Am. Chem. Soc. 2006, 128, 2115-2120. [CrossRef] [PubMed]

16. Haynes, C.L.; McFarland, A.D.; Van Duyne, R.P. Surface-enhanced raman spectroscopy. Anal. Chem. 2005, 77, 338a-346a. [CrossRef]

17. Wei, H.; Hao, F.; Huang, Y.; Wang, W.; Nordlander, P.; Xu, H. Polarization dependence of surface-enhanced raman scattering in gold nanoparticle-nanowire systems. Nano Lett. 2008, 8, 2497-2502. [CrossRef]

18. Tao, A.; Kim, F.; Hess, C.; Goldberger, J.; He, R.; Sun, Y.; Xia, Y.; Yang, P. Langmuir-blodgett silver nanowire monolayers for molecular sensing using surface-enhanced raman spectroscopy. Nano Lett. 2003, 3, 1229-1233. [CrossRef]

19. Dasary, S.S.; Singh, A.K.; Senapati, D.; Yu, H.; Ray, P.C. Gold nanoparticle based label-free SERS probe for ultrasensitive and selective detection of trinitrotoluene. J. Am. Chem. Soc. 2009, 131, 13806-13812. [CrossRef] [PubMed]

20. Jin, L.; She, G.; Li, J.; Xia, J.; Wang, X.; Mu, L.; Shi, W. A facile fabrication of ag-au-ag nanostructures with nanogaps for intensified surface-enhanced raman scattering. Appl. Surf. Sci. 2016, 389, 67-72. [CrossRef]

21. Sonnichsen, C.; Reinhard, B.M.; Liphardt, J.; Alivisatos, A.P. A molecular ruler based on plasmon coupling of single gold and silver nanoparticles. Nat. Biotechnol. 2005, 23, 741-745. [CrossRef] [PubMed]

22. Mock, J.J.; Hill, R.T.; Degiron, A.; Zauscher, S.; Chilkoti, A.; Smith, D.R. Distance-dependent plasmon resonant coupling between a gold nanoparticle and gold film. Nano Lett. 2008, 8, 2245-2252. [CrossRef] [PubMed]

23. Dill, T.J.; Rozin, M.J.; Brown, E.R.; Palani, S.; Tao, A.R. Investigating the effect of ag nanocube polydispersity on gap-mode SERS enhancement factors. Analyst 2016, 141, 3916-3924. [CrossRef] [PubMed]

24. Zhao, F.; Zeng, J.; Parvez Arnob, M.M.; Sun, P.; Qi, J.; Motwani, P.; Gheewala, M.; Li, C.H.; Paterson, A.; Strych, U.; et al. Monolithic NPG nanoparticles with large surface area, tunable plasmonics, and high-density internal hot-spots. Nanoscale 2014, 6, 8199-8207. [CrossRef] [PubMed]

25. Qi, J.; Zeng, J.; Zhao, F.; Lin, S.H.; Raja, B.; Strych, U.; Willson, R.C.; Shih, W.C. Label-free, in situ SERS monitoring of individual DNA hybridization in microfluidics. Nanoscale 2014, 6, 8521-8526. [CrossRef] [PubMed]

26. Santos, G.M.; Zhao, F.; Zeng, J.; Li, M.; Shih, W.C. Label-free, zeptomole cancer biomarker detection by surface-enhanced fluorescence on nanoporous gold disk plasmonic nanoparticles. J. Biophotonics 2015, 8, 855-863. [CrossRef] [PubMed]

27. Qiu, S.; Zhao, F.; Zenasni, O.; Li, J.; Shih, W.C. Nanoporous gold disks functionalized with stabilized g-quadruplex moieties for sensing small molecules. ACS Appl. Mater. Interfaces 2016, 8, 29968-29976. [CrossRef] [PubMed]

28. Li, M.; Du, Y.; Zhao, F.; Zeng, J.; Mohan, C.; Shih, W.C. Reagent- and separation-free measurements of urine creatinine concentration using stamping surface enhanced raman scattering (S-SERS). Biomed. Opt. Express 2015, 6, 849-858. [CrossRef] [PubMed] 
29. Santos, G.M.; Ferrara, F.I.d.S.; Zhao, F.; Rodrigues, D.F.; Shih, W.-C. Photothermal inactivation of heat-resistant bacteria on nanoporous gold disk arrays. Opt. Mater. Express 2016, 6, 1217. [CrossRef]

30. Zeng, J.; Zhao, F.; Qi, J.; Li, Y.; Li, C.-H.; Yao, Y.; Leebc, T.R.; Shih, W.-C. Internal and external morphology-dependent plasmonic resonance in monolithic nanoporous gold nanoparticles. RSC Adv. 2014, 4, 36682-36688. [CrossRef]

31. Arnob, M.M.; Zhao, F.; Zeng, J.; Santos, G.M.; Li, M.; Shih, W.C. Laser rapid thermal annealing enables tunable plasmonics in nanoporous gold nanoparticles. Nanoscale 2014, 6, 12470-12475. [CrossRef] [PubMed]

32. Zeng, J.; Zhao, F.; Li, M.; Li, C.-H.; Lee, T.R.; Shih, W.-C. Morphological control and plasmonic tuning of nanoporous gold disks by surface modifications. J. Mater. Chem. C 2015, 3, 247-252. [CrossRef]

33. Boyd, D.A.; Bezares, F.J.; Pacardo, D.B.; Ukaegbu, M.; Hosten, C.; Ligler, F.S. Small-molecule detection in thiol-yne nanocomposites via surface-enhanced raman spectroscopy. Anal. Chem. 2014, 86, 12315-12320. [CrossRef] [PubMed]

34. Qi, J.; Shih, W.C. Performance of line-scan raman microscopy for high-throughput chemical imaging of cell population. Appl. Opt. 2014, 53, 2881-2885. [CrossRef] [PubMed]

35. Wang, D.; Schaaf, P. Nanoporous gold nanoparticles. J. Mater. Chem. 2012, 22, 5344. [CrossRef]

36. Vidal, C.; Wang, D.; Schaaf, P.; Hrelescu, C.; Klar, T.A. Optical plasmons of individual gold nanosponges. ACS Photonics 2015, 2, 1436-1442. [CrossRef] [PubMed]

37. Arnob, M.M.P.; Zhao, F.; Li, J.; Shih, W.-C. New fabrication and modeling techniques for nanoporous gold nanodisks. ACS Photonics 2017. [CrossRef]

38. Enustun, B.V.; Turkevich, J. Coagulation of colloidal gold. J. Am. Chem. Soc. 1963, 85, 3317-3328. [CrossRef]

39. Kimling, J.; Maier, M.; Okenve, B.; Kotaidis, V.; Ballot, H.; Plech, A. Turkevich method for gold nanoparticle synthesis revisited. J. Phys. Chem. B 2006, 110, 15700-15707. [CrossRef] [PubMed]

40. Boyd, D.A.; Naciri, J.; Fontana, J.; Pacardo, D.B.; Shields, A.R.; Verbarg, J.; Spillmann, C.M.; Ligler, F.S. Facile fabrication of color tunable film and fiber nanocomposites via thiol click chemistry. Macromolecules 2014, 47, 695-704. [CrossRef]

41. Qian, L.; Das, B.; Li, Y.; Yang, Z. Giant raman enhancement on nanoporous gold film by conjugating with nanoparticles for single-molecule detection. J. Mater. Chem. 2010, 20, 6891. [CrossRef]

42. Cai, H.; Zhu, J.; Chen, G.; Liu, L.; He, G.S.; Zhang, X. Surface-enhanced raman scattering and dft calculations studies of 3,3'-diethylthiatri- carbocyanine iodide. J. Raman Spectrosc. 2011, 42, 1722-1727. [CrossRef] 